\title{
CONDICIONES MICROCLIMÁTICAS, HÁBITOS DE PERCHA Y ESPECIES ASOCIADAS A LOS REFUGIOS DE NATALUS STRAMINEUS EN MÉXICO
}

\author{
José Williams TORRES-FLORES y Ricardo LÓPEZ-WILCHIS \\ Departamento de Biología, Universidad Autónoma Metropolitana, Unidad Iztapalapa, Apdo. Postal 55-535, \\ CP 09340, D.F. MÉXICO. E-mail: bravadotf@yahoo.com.mx, rlw@xanum.uam.mx
}

Torres-Flores, J.W. \& R. López-Wilchis. 2010. Condiciones microclimáticas, hábitos de percha y especies asociadas a los refugios de Natalus stramineus en México. Acta Zoológica Mexicana (n.s.), 26(1): 191-213.

RESUMEN. Natalus stramineus es un murciélago cavernícola ampliamente extendido en México del que poco se sabe sobre su historia natural. En el presente trabajo, se reportan los refugios y las especies asociadas a este murciélago en México. Además se reporta la variación mensual de la temperatura ambiente y humedad relativa en una cueva donde se alberga, localizada en Colima, México, donde también se observaron sus hábitos de percha. Este murciélago ha sido encontrado en 108 refugios que en su gran mayoría son cuevas y minas, los cuales se localizaron en un amplio intervalo de altitud, tipos de vegetación y climas; sin embargo, la mayoría se encuentran a alturas menores a los $400 \mathrm{msnm}$, en selva baja caducifolia y con clima cálido-subhúmedo. En algunos sitios donde se había reportado su presencia no pudo ser encontrado durante los muestreos realizados. En la cueva El Salitre, la temperatura y humedad en sus sitios de percha permanecieron estables a lo largo del año. La primera variable osciló de 25.8 a $26.9^{\circ} \mathrm{C}$ y la segunda de 92.1 a $100 \%$. Los individuos usualmente se cuelgan en las partes bajas de las paredes y en techos inclinados, utilizando también cavidades de disolución y ocasionalmente grietas. Perchan individualmente sin estar en contacto directo uno con otro, manteniendo una distancia de $5-50 \mathrm{~cm}$. Se registraron 45 especies de murciélagos asociadas a $N$. stramineus, pero con mayor frecuencia a Pteronotus parnellii, Mormoops megalophylla, Glossophaga soricina y Desmodus rotundus. N. stramineus prefiere ocupar secciones cálidas y muy húmedas de los refugios, siendo estas condiciones ideales para conservar el calor corporal y mantener en buen estado sus alas. Su gran tendencia a asociarse con mormópidos y algunos filostómidos se explica, en parte, por requerir condiciones microclimáticas similares en sus refugios, así como por los beneficios termoregulatorios que tiene el agrupamiento en colonias. Este murciélago depende mucho de cuevas de calor para su supervivencia y ocupa de manera oportunista minas con características microclimáticas similares.

Palabras clave: Natalus stramineus, condiciones microclimáticas, hábitos de percha, México.

Torres-Flores, J.W. \& R. López-Wilchis. 2010. Microclimatic conditions, roosting habits, and associated species to refuges of Natalus stramineus in Mexico. Acta Zoológica Mexicana (n.s.), 26(1): 191-213.

ABSTRACT. Natalus stramineus is a cave-dwelling bat broadly spread in Mexico but with scarce information about its natural history. In this work, we reported the associated bat species and roosting sites of $N$. stramineus in Mexico. Also we observed roosting habits and recorded monthly air

Recibido: 16/06/2009; aceptado: 10/02/2010. 
temperature and relative humidity throughout one year in a roost of this bat, located in the state of Colima, Mexico. It has been found in 108 roosting sites most of which are caves and mines. These roosts were located in a wide range of elevations, vegetation and climates, however most were located below $400 \mathrm{~m}$ asl, in tropical deciduous forest and hot-subhumid conditions. We were unable to find this species in some previously recorded caves. In El Salitre Cave, temperature and relative humidity in their roosting sites were stable throughout the year. The former variable fluctuates from 25.8 to $26.9^{\circ} \mathrm{C}$ and the latter variable from 92.1 to $100 \%$. Individuals of $N$. stramineus usually hang in the lower parts of walls and rocky ceilings, occupying cavities and crevices occasionally. They hang individually without contact to each other, keeping a distance of $5-50 \mathrm{~cm}$ between themselves. Forty-five bat species have been found associated with this natalid, but more frequently Pteronotus parnellii, Mormoops megalophylla, Glossophaga soricina y Desmodus rotundus. This bat prefers to occupy warm and humid parts of the roosts, because these conditions are ideal to conserve the body heat and to maintain their wings in good conditions. Its great tendency to be associated with mormopids and some phyllostomids is explained partly by their similar microclimatic requirements in their refuges, as well as by the thermal benefits associated with clustering in colonies. N. stramineus depends heavily on hot caves for their survivorship and occupies mines with similar microclimatic conditions in an opportunistic way.

Key words: Natalus stramineus, roosts, microclimatic conditions, roosting habits, Mexico.

\section{INTRODUCCIÓN}

Natalus stramineus Gray, 1838, es un murciélago perteneciente a la familia Natalidae, cuyos miembros son insectívoros de tamaño pequeño (5-8 g) restringidos exclusivamente a la región Neotropical (Dalquest 1950; Emmons 1997; Goodwin 1959; Simmons 2005; Tejedor et al. 2005). Esta especie se distribuye desde el norte de México hasta al este de Paraguay y Bolivia, ocupando además parte de las Antillas (Reid 1997; Simmons 2005; Taddei \& Uieda 2001). Es un murciélago gregario que utiliza generalmente cuevas como refugios diurnos, tendiendo a ocuparlos junto con otras especies de quirópteros (Arita 1993; Taddei \& Uieda 2001). Al igual que otros natálidos, depende fuertemente de la presencia de estos sitios para garantizar su supervivencia, determinando en gran parte, su distribución y abundancia (Emmons 1997).

A pesar de estar ampliamente distribuido y de ser conocido desde hace tiempo, poco se sabe sobre su historia natural. Solo se tienen datos básicamente anecdóticos sobre algunos aspectos reproductivos (Krutzch 2000; Sánchez-Hernández et al. 2002), fisiológicos (Ávila-Flores \& Medellín 2004) y poblacionales (Mitchell 1967), así como de su morfología alar (Jennings et al. 2004; Norberg \& Rayner 1987), velocidad de vuelo (Akins et al. 2007) y sonidos de ecolocación (Jennings et al. 2004; Rydell et al. 2002).

Para nuestro país contamos con una gran cantidad de registros que documentan su presencia, aunque la mayoría de ellos tiene más de 30 años de antigüedad (LópezWilchis 2003). Poco sabemos sobre si esas poblaciones continúan hasta la actualidad y menos aun de las características de los refugios, de su entorno circundante y de los requerimientos microclimáticos de esta especie para permanecer en los mismos. 
Por otra parte, la selección de los sitios de percha así como de condiciones microclimáticas adecuadas es de vital importancia entre los murciélagos, ya que pasan la mayor parte del día descansando en sus refugios diurnos (Kunz 1982). El conocimiento de las características microclimáticas en dichos sitios, tales como la temperatura ambiente y humedad relativa, es relevante ya que estos factores abióticos influyen de forma importante en la selección de los refugios pues afectan las tasas metabólicas, la termorregulación e influyen en el desarrollo de los embriones y crías (Speakman \& Thomas 2003; Tuttle \& Stevenson 1982). Su importancia se ha manifestado en varias especies de murciélagos que muestran preferencias por temperaturas y/o humedades específicas en sus sitios de descanso (e.g. Baudinette et al. 2000; Kerth et al. 2001; Rodríguez-Durán \& Soto-Centeno 2003).

En sitios relativamente amplios y estructuralmente complejos, como cuevas y minas, suelen encontrarse varias especies de murciélagos coexistiendo ya sea de manera temporal o permanente, formando a menudo colonias muy numerosas en su interior sobre todo en zonas tropicales (Arita 1993; Graham 1988; Rodríguez-Durán 1998). En México hay un gran número de cuevas y minas, muchas de las cuales sirven como refugio principal o alternativo para casi la mitad de las especies de murciélagos que se encuentran en nuestro país (Arita 1993). No obstante, en muchos de estos lugares aun no se ha registrado la quiropterofauna que albergan, ni sus parámetros microambientales y tampoco sus variaciones poblacionales a largo plazo.

Los objetivos del presente trabajo fueron: 1) caracterizar los refugios utilizados por $N$. stramineus a lo largo de su distribución en nuestro país, así como el tipo de clima y vegetación circundante; 2) confirmar su presencia en sitios reportados previamente con más de 30 años de antigüedad y considerados como importantes por su posición geográfica y/o por el número de organismos reportados; 3 ) determinar las condiciones microclimáticas en los sitios de descanso de $N$. stramineus en una cueva del oeste de México; 4) describir sus hábitos de percha y 5) documentar las especies asociadas a este murciélago en México.

\section{MATERIAL Y MÉTODOS}

La caracterización de los refugios se realizó mediante una exhaustiva búsqueda bibliográfica, con la consulta de bases de datos (CNMA 2007; López-Wilchis 2003) y mediante la toma de los parámetros in situ en varios refugios localizados a lo largo de su distribución. Algunos registros bibliográficos fueron georreferenciados con la ayuda del programa Google Earth 2008 (ver. 4.3.7284.3916). El tipo de clima existente en la zona circundante a cada refugio se determinó consultando las Cartas Climatológicas escala 1:1,000,000 y la vegetación circundante mediante las Cartas de Vegetación y Uso del Suelo escala 1:250,000 de cada entidad federativa (INEGI 2009). 
Para confirmar su presencia en refugios considerados como relevantes por su posición geográfica y/o por el número de organismos que estaban presentes, con base en la información recabada se escogieron 15 sitios con más de 30 años de haber sido reportados para ser visitados por nosotros (Apéndice 1). Los muestreos se llevaron a cabo en diferentes meses y años: marzo del 2004, febrero del 2005, agosto del 2007, marzo-abril del 2008 y marzo-abril del 2009. En la medida de lo posible, en estas visitas se realizaron incursiones en los refugios para estimar visualmente el número de organismos presentes; cuando no fue posible hacerlo, la estimación se hizo mediante la captura de organismos utilizando redes entomológicas, redes de niebla y trampas de arpa según fuera el caso. Las coordenadas y altura de cada sitio fueron registradas utilizando un GPS Garmin map-60CSx.

Por ser un refugio estructuralmente complejo y por facilidades logísticas, se escogió la cueva El Salitre, localizada en el estado de Colima (Fig. 1, Apéndice 1) para estudiar tanto las condiciones microclimáticas que requiere esta especie como sus hábitos de percha. La cueva tiene dos entradas, ambas menores a los $2 \mathrm{~m}$ de altura y ancho. El clima en la zona es cálido-subhúmedo, con un promedio anual de precipitación de $738 \mathrm{~mm}$ y promedio anual de temperatura mayor a $\operatorname{los} 25^{\circ} \mathrm{C}$ (Estación Climatológica Los Ortices, Colima, Servicio Meteorológico Nacional). La temporada de lluvias abarca el periodo junio-septiembre y la temporada de secas comprende octubre-mayo. En mayo y junio del 2005, se realizaron exploraciones en la cueva para elaborar el mapa topográfico, ubicar las colonias de $N$. stramineus y de las especies asociadas, y definir los sitios para la toma de los parámetros ambientales. Las dimensiones se estimaron usando una cinta métrica, mientras que la orientación y pendiente fueron determinadas con una brújula Brunton 5006 LMS. De abril de 2006 a marzo de 2007 se registró mensualmente la temperatura ambiente y humedad relativa con un data logger Hobo H8 Pro Series (Onset Computer Corp.) colocado en un pasaje de la cueva donde se albergan cientos de individuos de $N$. stramineus. Ambas variables fueron registradas de manera automática cada 5 minutos durante dos días continuos en cada muestreo mensual. Asimismo, con otro data logger colocado 10 metros afuera de la entrada se registraron la temperatura ambiente y humedad relativa cada 3 minutos durante dos noches continuas. Para determinar si existían diferencias en la temperatura y humedad en los sitios de percha tanto entre meses como entre temporadas, se efectuaron las pruebas no paramétricas de Kruskal-Wallis y U de Mann-Whitney respectivamente, determinando la significancia estadística a $\leq 0.05$. El análisis se llevo a cabo utilizando el programa Stata versión 8.0 (Stata Corp. 2003). Los hábitos de percha fueron observados y registrados visualmente en un área específica de la cueva, completando con otras observaciones realizadas durante las incursiones a otros refugios. 
Las especies asociadas a este murciélago fueron reconocidas con base en los registros obtenidos directamente por nosotros y mediante la literatura consultada. Para la determinación in situ se siguieron las claves de Medellín et al. (1997), siguiendo a Ramírez-Pulido et al. (2005) para su nomenclatura (excepto Leptonycteris yerbabuenae).

Como es probable que en reportes previos en algunas localidades los individuos referidos como $N$. stramineus hayan correspondido a $N$. lanatus, especie recientemente descrita en México (Tejedor 2005) y debido a la imposibilidad de precisar su identidad taxonómica, toda la información recabada para este estudio fue incluida en el análisis como perteneciente a $N$. stramineus. Asimismo, aunque se ha propuesto la denominación de $N$. mexicanus para las poblaciones de $N$. stramineus con distribución en nuestro país (Tejedor 2006), se requiere de más evidencias al respecto, por lo que en este trabajo mantenemos el nombre específico de stramineus en tanto no se clarifiquen estos aspectos.

\section{RESULTADOS}

Caracterización de los refugios. Después de depurar los datos bibliográficos y mediante el trabajo de campo realizado por nosotros, logramos integrar un total de 310 registros de ocurrencia para $N$. stramineus en nuestro país, de los cuales 108 hacen mención a sus refugios (Apéndice 1). Para la toma de diversos parámetros que nos permitieron caracterizar sus refugios visitamos 36 sitios, entre los que se incluyen 12 que no habían sido reportados previamente (Fig. 1). Muchos de estos sitios se encuentran en los estados del sur y norte del país, siendo Yucatán y Tamaulipas respectivamente donde más se han registrado. Por otro lado, la mayoría de las minas donde se ha encontrado se localizan en los estados de Baja California Sur, Sonora, Sinaloa y Chihuahua.

La gran mayoría de los refugios que utiliza esta especie corresponden a cuevas (70\%), ocupando con menor frecuencia las minas (23\%) y ocasionalmente otro tipo de estructuras como túneles $(6 \%)$ o alcantarillas $(1 \%)$. En términos generales, estos sitios se encuentran localizados cerca de ríos y arroyos o presentan cuerpos de agua en su interior, no obstante algunos se localizaron en zonas semidesérticas (e.g. Mina Estrella Polar, en Baja California Sur).

En general, las cuevas utilizadas son estructuralmente muy complejas, presentando una o varias entradas, con varias cámaras y pasajes de dimensiones muy variables, con paredes rugosas, grietas y oquedades; con zonas donde hay una amplia circulación del viento y otras donde no circula el aire y generalmente saturadas de amoniaco. La mayoría están constituidas por formaciones calizas, pero también hay algunas formadas por estratos metamórficos y basálticos. Su longitud es muy variable, desde algunos metros (Colotlán, Michoacán), hasta algunas con varios kilómetros o cuya longitud total aún no ha sido determinada (San Sebastián de las 


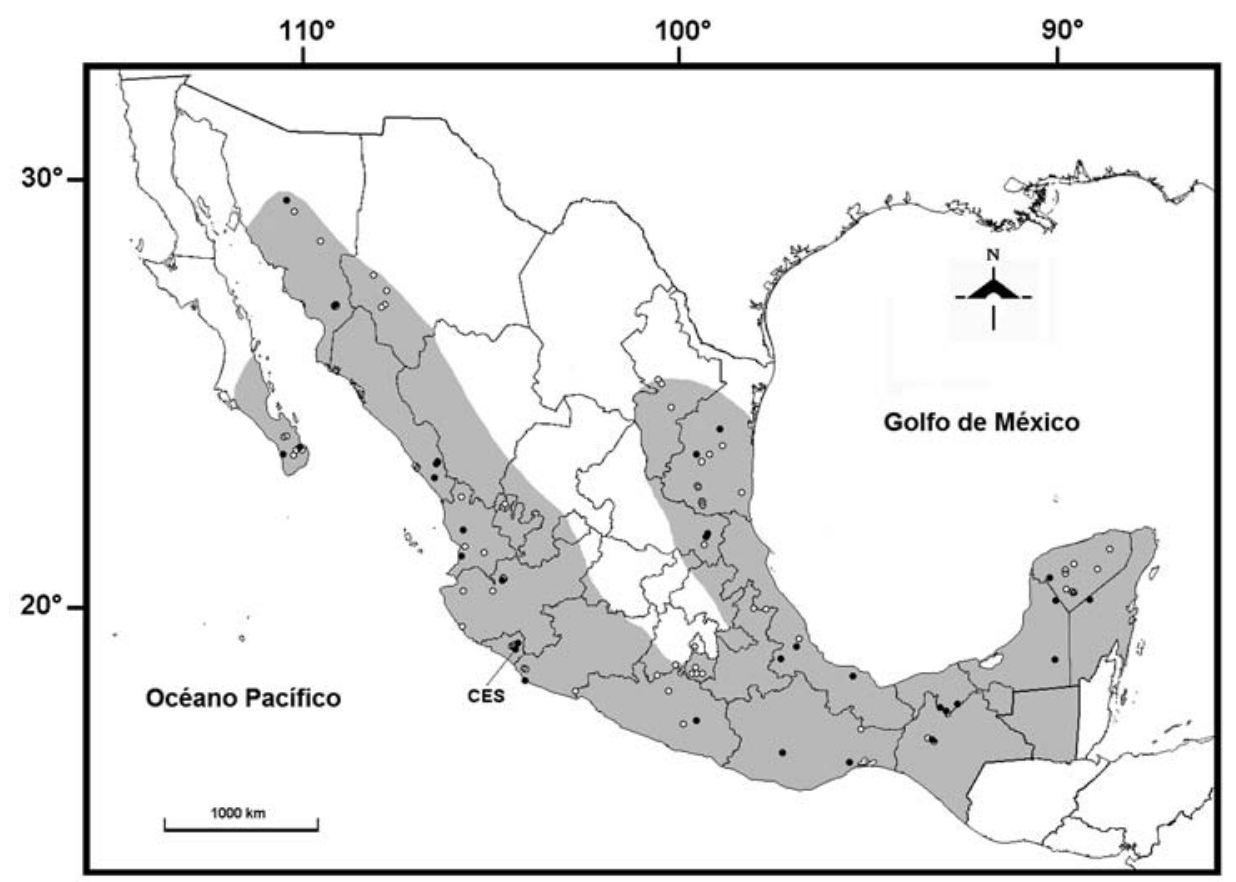

Figura 1. Refugios de $N$. stramineus muestreados en nuestro estudio $(\bullet)$ y reportados en la bibliografía $\left(^{\circ}\right)$. El área sombreada representa su distribución geográfica en México (modificada de Medellín et al. 1997). CES = Cueva "El Salitre", Colima.

Grutas, Oaxaca). Los túneles corresponden a estructuras de concreto o ladrillo, de algunos metros de ancho y altura, excepcionalmente con más de $200 \mathrm{~m}$ de longitud; sus paredes son muy húmedas y tienen agua en el piso.

Durante nuestros recorridos encontramos sitios desde nivel del mar (Colotlán, Michoacán) hasta los 1709 msnm (San Sebastián de las Grutas, Oaxaca). Más de la mitad de los refugios (54\%) se ubican a menos de $400 \mathrm{~m}$, un $22 \%$ se localizan entre los 400 y 800 , un $13 \%$ entre los 801 y los 1200 y $11 \%$ están por arriba de los 1200 msnm. La vegetación circundante a estos sitios es variada (Fig. 2), teniendo claramente las selvas caducifolias el mayor porcentaje de ocurrencia (68\%), seguida de matorral subtropical o xerófito (12\%), selvas altas perennifolias $(12 \%)$ y bosques de pino-encino o mesófilos (8\%). El clima imperante en la zona donde se localizó cada refugio también es variable, desde los secos-muy cálidos hasta los semifríossubhúmedos, aunque la mayoría corresponden a los cálidos-subhúmedos o subcálidos-subhúmedos (en $66 \%$ de los sitios).

Los individuos siempre se encontraron en las partes profundas, más oscuras y generalmente más húmedas de las cuevas y minas. En nuestras incursiones 


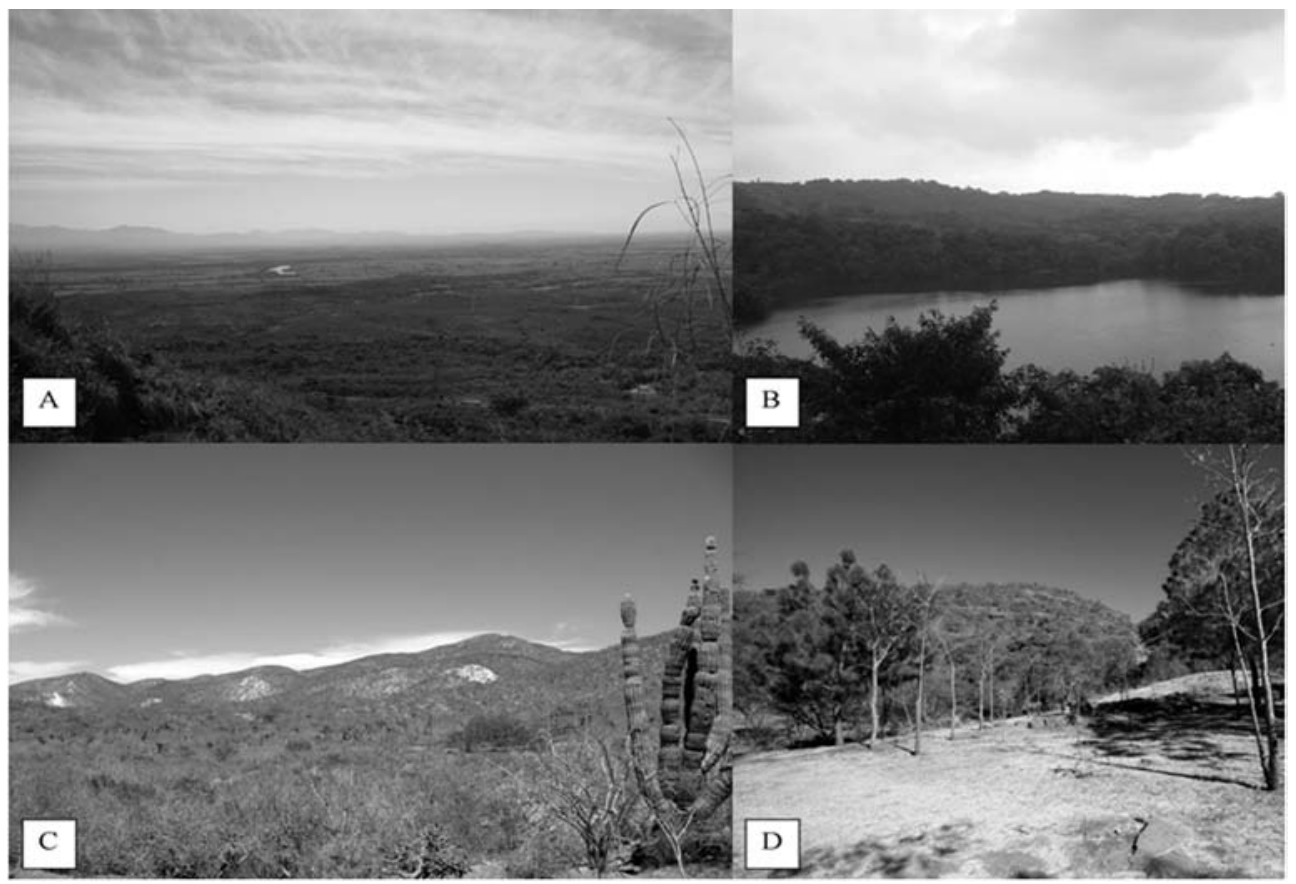

Figura 2. Fotografías de la vegetación circundante en algunos refugios de $N$. stramineus visitados en nuestro estudio. (A) Cueva en "Cerro de Las Viejas", Santiago Ixcuintla, Nayarit; (B) Cueva "El Socavón”, San Andrés Tuxtla, Veracruz; (C) Mina "Estrella Polar”, Pescadero, Baja California Sur y; (D) Cueva en San Sebastián de las Grutas, Oaxaca.

registramos que la humedad relativa siempre fue superior al $70 \%$ y con temperatura ambiente entre los 25 y $30^{\circ} \mathrm{C}$. Solamente en "Arroyo del Bellaco" y en "Los Laguitos" registramos temperaturas muy cálidas $\left(32.5\right.$ y $34.5^{\circ} \mathrm{C}$ respectivamente), mientras que en "El Amparo" y "San Sebastián de las Grutas" registramos temperaturas templadas $\left(20 \mathrm{y} 21^{\circ} \mathrm{C}\right.$ respectivamente).

Número de individuos y permanencia en los refugios. El número de organismos presentes en los refugios varió ampliamente, desde lugares en los que no estaban presentes pero en los cuales se había reportado previamente su presencia, hasta grandes colonias formadas por más de 5,000 individuos. La mayoría de las colonias observadas por nosotros tenían menos de 100 ó 200 individuos, 7 estaban cercanas a los 500 y solamente 3 presentaron más de 1,000 individuos. En la cueva "Río Escondido", Tamaulipas, el numero de individuos presentes fue mayor al reportado en la literatura; en la cueva de Juxtlahuaca, Guerrero, el tamaño poblacional coincidió con los reportes anteriores; pero en el resto de los lugares por lo general el número fue menor al documentado en la literatura, incluso en 10 refugios no se encontraron ejemplares (Apéndice 1). 
Condiciones microclimáticas. En la cueva "El Salitre", Colima, los datos mostraron que la temperatura ambiente y la humedad relativa en los sitios de percha permanecieron relativamente estables a lo largo del año (Fig. 3a). La primera varió entre 25.8 y $26.9^{\circ} \mathrm{C}(\mathrm{n}=6905, \overline{\mathrm{X}}=26.2$, d. e. $=0.2)$, presentando diferencias significativas entre meses (Kruskal-Wallis $\chi^{2}=6224$, g. $1 .=11, \mathrm{P}<0.001$ ) y entre temporadas (Mann-Whitney $\mathrm{z}=-40.86, \mathrm{P}<0.001$ ) siendo mayor en la de lluvias. La segunda varió entre 92.1 y $100 \%(\mathrm{n}=6905, \mathrm{X}=99.3$, d. e. $=1.7)$ con diferencias significativas entre meses $\left(\chi^{2}=3484\right.$, g. $\left.1 .=11, \mathrm{P}<0.001\right)$ sobre todo en abril y mayo del 2006, aunque no existieron diferencias entre temporadas $(z=0.46, P=0.64)$. En contraste, la temperatura y humedad externas fueron muy variables en cada mes y a lo largo del año (Fig. 3b). La primera osciló de 13.3 a $31.1^{\circ} \mathrm{C}(\mathrm{n}=4430, \bar{X}=22.9$, d. e. $=2.9)$ y la segunda de 23.2 a $81.9 \%(n=4430, \bar{X}=40.1$, d. e. $=11.5)$.
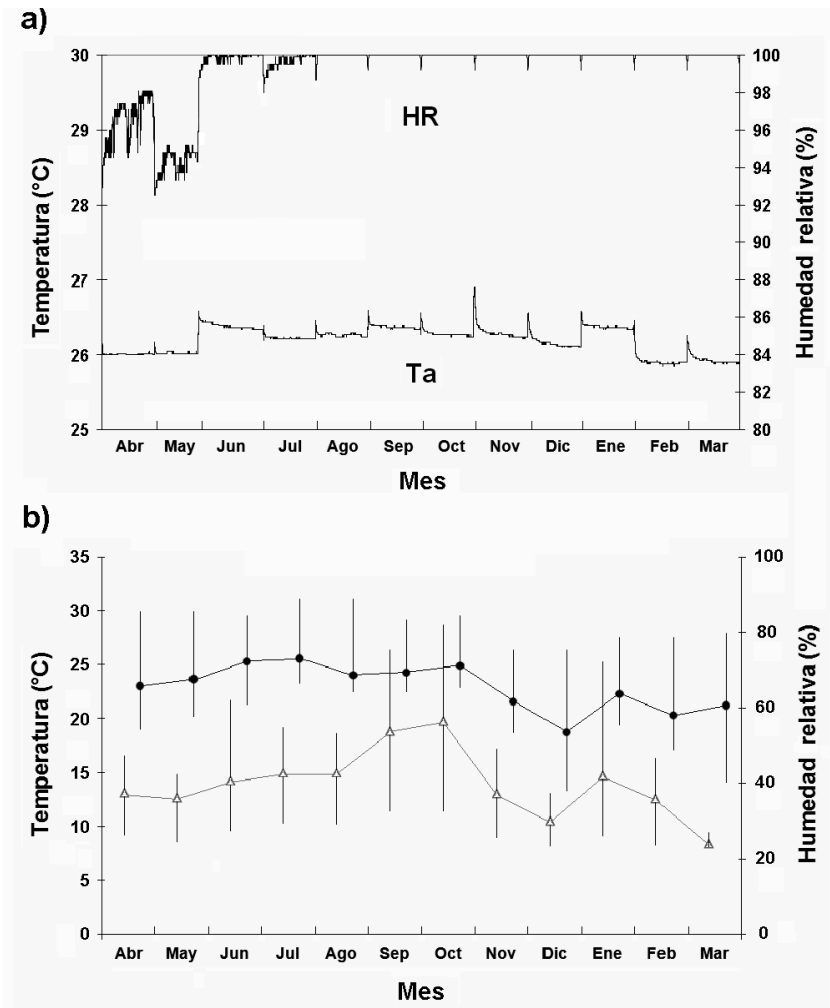

Figura 3. a) Temperatura ambiental (Ta) y humedad relativa (HR) registradas mensualmente en áreas de percha de N. stramineus en la cueva El Salitre durante el periodo abril 2006-marzo 2007.

b) Promedio, máximo y mínimo de la Ta $(\bullet-)$ y HR $(\triangle)$ externas registradas en un área adyacente a la cueva durante el mismo periodo. 
Hábitos de percha. En la cueva El Salitre encontramos descansando a $N$. stramineus en las secciones más profundas, siempre a una distancia $>80 \mathrm{~m}$ de la entrada y en condiciones de oscuridad total (Fig. 4). Observamos que los individuos usualmente perchan en las partes bajas de las paredes y en techos inclinados ( $<4 \mathrm{~m}$ de altura), así como en cavidades de disolución y ocasionalmente en grietas. Descansan muy a menudo sin entrar en contacto ventral con el sustrato, sujetándose ya sea con una o con las dos patas. Se cuelgan individualmente sin estar en contacto directo uno con otro, manteniendo una distancia que varía de 5 a $50 \mathrm{~cm}$. En la época de reproducción las hembras forman colonias de maternidad apartadas de los machos, agrupándose en racimos. Estos aspectos conductuales también fueron registrados en todos los refugios visitados.

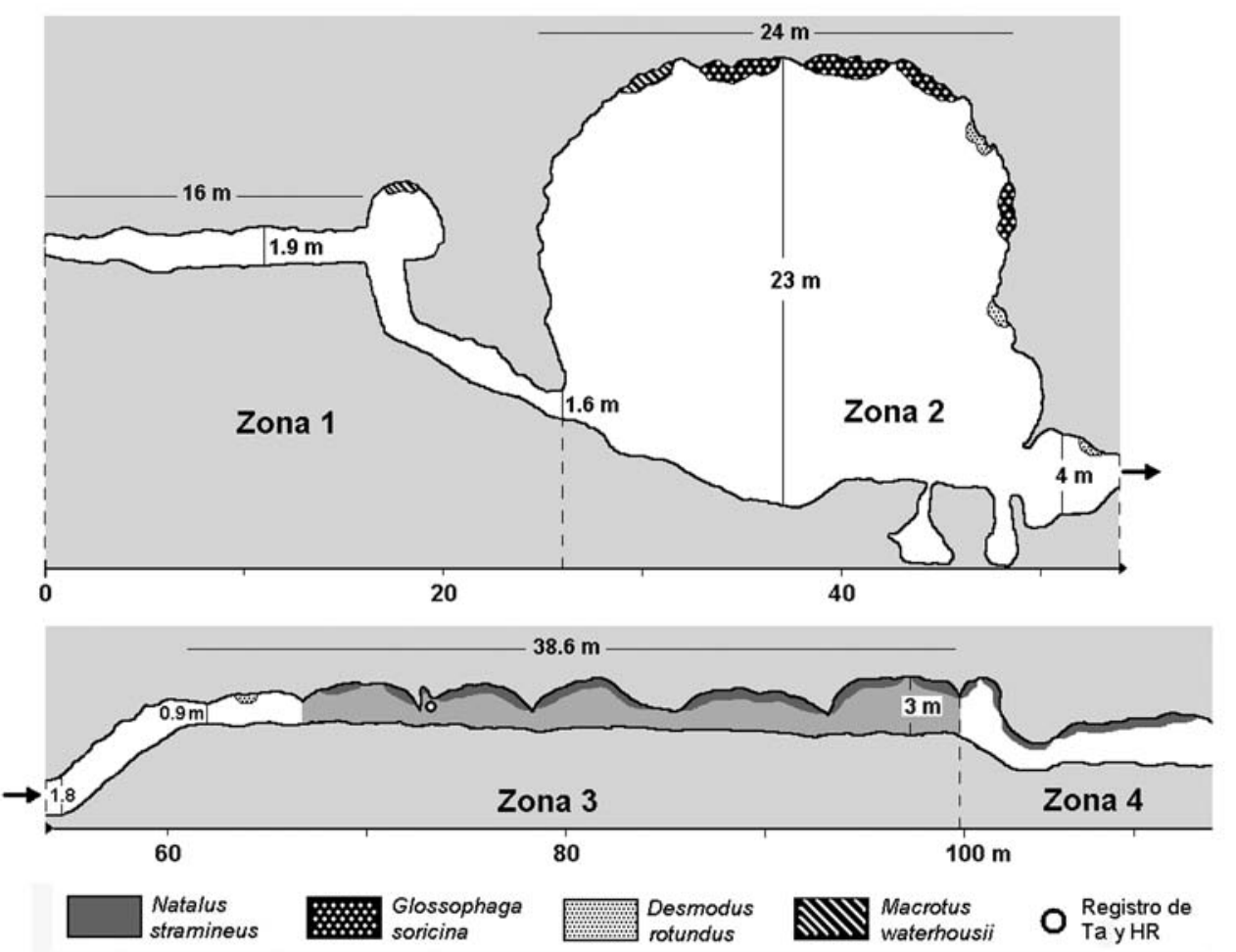

Figura 4. Perfil topográfico y dimensiones de la cueva El Salitre, Colima. El esquema muestra el perfil desde la entrada más chica de la cueva hasta la última sección que pudo ser explorada. La parte sombreada representa las áreas donde se observaron los hábitos de percha de N. stramineus. 
Especies asociadas. Se han registrado 45 especies de murciélagos asociadas a $N$. stramineus en México: 28 filostómidos, 5 mormópidos, 7 vespertiliónidos, 3 molósidos y 2 embalónuridos (Cuadro 1). Las especies que se encontraron asociadas con mayor frecuencia fueron los mormópidos Pteronotus parnellii (en $47 \%$ de los sitios), Mormoops megalophylla (32\%), P. davyi (27\%) y P. personatus (17\%); además de los filostómidos Glossophaga soricina (37\%), Desmodus rotundus (34\%) y Artibeus jamaicensis (19\%). Varias especies no cavernícolas (e. g. Glyphonycteris sylvestris) se han encontrado ocasionalmente con esta especie. En los muestreos realizados por distintas cuevas del país, registramos asociadas por primera vez a 6 especies (Cuadro 1). En el $25 \%$ de los refugios $N$. stramineus se registró solitariamente, en el $38 \%$ en asociación con 1-3 especies y en $37 \%$ con $\geq 4$ especies.

Cuadro 1. Especies asociadas y número de sitios registrados en asociación con $N$. stramineus en México. Las especies se enlistan por familia.

\begin{tabular}{|c|c|c|c|}
\hline Especies & No. sitios & Especies & No. sitios \\
\hline$\overline{\text { Phyllostomidae }}$ & & Mormoopidae & \\
\hline Anoura geoffroyi & 2 & Mormoops megalophylla & 34 \\
\hline Artibeus hirsutus & 4 & Pteronotus davyi & 29 \\
\hline A. intermedius & 3 & P. gymnonotus * & 2 \\
\hline A. jamaicensis & 20 & P. parnellii & 50 \\
\hline A. lituratus & 4 & P. personatus & 18 \\
\hline Carollia perspicillata & 7 & & \\
\hline C. sowelli $*$ & 1 & Vespertilionidae & \\
\hline C. subrufa & 1 & Corynorhinus mexicanus $*$ & 1 \\
\hline Choeronycteris mexicana & 2 & Myotis auriculus * & 1 \\
\hline Chrotopterus auritus & 1 & M. keaysi & 9 \\
\hline Dermanura azteca* & 1 & M. nigricans & 2 \\
\hline D. phaeotis & 1 & M. velifer & 9 \\
\hline D. tolteca & 1 & M. yumanensis & 1 \\
\hline Desmodus rotundus & 36 & Parastrellus hesperus & 1 \\
\hline Diphylla ecaudata & 10 & & \\
\hline Glossophaga leachii & 6 & Molossidae & \\
\hline G. morenoi & 1 & Nyctinomops aurispinosus & 1 \\
\hline G. soricina & 40 & N. laticaudatus & 1 \\
\hline Glyphonycteris sylvestris & 1 & Tadarida brasiliensis & 6 \\
\hline Leptonycteris nivalis & 10 & & \\
\hline L. yerbabuenae & 13 & Emballonuridae & \\
\hline Lonchorhina aurita * & 1 & Balantiopteryx plicata & 10 \\
\hline Macrotus californicus & 3 & Peropteryx macrotis & 6 \\
\hline M. waterhousii & 11 & & \\
\hline Micronycteris microtis & 1 & & \\
\hline Mimon bennettii & 1 & & \\
\hline Sturnira lilium & 3 & & \\
\hline S. ludovici & 1 & & \\
\hline
\end{tabular}

\footnotetext{
* Nuevos registros como especies asociadas.
} 
En la cueva El Salitre, Colima, pudimos observar que varios ejemplares de $N$. stramineus se ven desplazados parcialmente por M. megalophylla en ciertos meses del año. En febrero del 2007 observamos que M. megalophylla ocupa gran parte de las áreas de percha utilizadas por $N$. stramineus; en marzo observamos también esta coexistencia, aunque el número de individuos de M. megalophylla fue mucho menor, al igual que el área que ocupaban. En abril este mormópido ya no se encontró en este pasaje, ni en otras partes de la cueva, ocupándolo exclusivamente $N$. stramineus el resto del año. Por otro lado, también llegamos a observar algunos individuos del vampiro $D$. rotundus ocupando algunas oquedades entre algunos grupos de $N$. stramineus. En ambos casos no vimos que los individuos de estas especies se mezclaran, ni tampoco alguna conducta agresiva entre ellos.

\section{DISCUSIÓN}

Natalus stramineus se comporta como una especie oportunista en la selección de los sitios que ocupa como refugios diurnos. A lo largo de su distribución ocupa con mayor frecuencia las cuevas, pero en los estados del noroeste utiliza varias minas abandonadas y en algunas ocasiones construcciones humanas como túneles y alcantarillas. Esto puede deberse a que en el noroeste del país haya tanto una menor cantidad de cuevas como una mayor disponibilidad de minas. Asimismo, sus refugios se localizan preferentemente desde el nivel del mar hasta los $400 \mathrm{msnm}$, pero los hay a más de $1700 \mathrm{msnm}$, abarcando varios tipos de vegetación y climas que van desde zonas semidesérticas con matorral xerófito y climas cálidos-secos hasta zonas templadas con bosques de pino-encino y clima templado-subhúmedo (Fig. 2). Estos datos sugieren que este murciélago tiene una amplia plasticidad para adaptarse a diferentes condiciones macroclimáticas. Sin embargo, podemos decir que se encuentra típicamente en refugios ubicados en tierras bajas, con vegetación de selva baja o mediana caducifolia y con clima cálido-subhúmedo o subcálido-subhúmedo.

En nuestras visitas, así como en reportes previos, se observó que $N$. stramineus conforma colonias pequeñas a medianas en tamaño, constituidas por algunas decenas o cientos de individuos y en raras ocasiones por algunos miles. En nuestras incursiones a varias cuevas y minas, no fue posible llegar hasta los lugares precisos donde se alberga este murciélago para hacer una estimación visual del tamaño de sus colonias, aunque nuestras capturas indican que en la mayoría de los casos estaban formadas por cientos de individuos. La colonia más grande que observamos fue localizada en marzo del 2004 en la cueva ubicada en "Rancho Río Escondido", Tamaulipas, donde existe una colonia de maternidad formada por más de 5,000 individuos. Visitamos la cueva "Los Laguitos" y la mina "Estrella Polar" donde se habían registrado colonias formadas por 10,000 individuos; la primera la recorrimos en su totalidad, pero en ambos casos nuestros registros fueron mucho menores. Además, en varios de los sitios donde se había reportado la presencia de esta especie no se localizaron ejemplares 
durante nuestros muestreos, lo que podría deberse a la alteración del sitio y/o a la realización de migraciones temporales. La cueva "Diana Liesa", Oaxaca, ha sido objeto de una profunda transformación y actualmente es usada como una iglesia y es poco probable que sea usada como refugio diurno; lo mismo puede estar ocurriendo en la cueva "El Socavón", Veracruz, que es visitada a diario por personas que dejan una gran cantidad de ofrendas y veladoras prendidas en su interior. En el caso de la cueva "El Tigre", "El Volcán de los Murciélagos" y varios refugios más que prácticamente no han sufrido cambio alguno, lo más probable es que su ausencia se deba a la realización de movimientos. Con base en las observaciones hechas en la cueva "El Salitre", Colima, pudimos constatar que esta especie no siempre está presente en el refugio a lo largo del año. Las fluctuaciones en su número son estacionales, debido con seguridad a la realización de movimientos locales hacia otras cuevas y/o a una segregación sexual durante la época reproductiva (Torres-Flores 2005). No obstante, con nuestros datos no se puede asegurar con certeza si el tamaño poblacional en sus refugios ha decaído en años recientes.

Los datos obtenidos en la cueva de estudio, así como en otros refugios indican claramente que $N$. stramineus prefiere ocupar aquellas secciones con temperaturas cálidas y humedad muy alta, con poca variación a lo largo del año. Las características físicas de estas secciones explican en parte la estabilidad de ambos parámetros, pues las colonias de esta especie siempre se encontraron en pasajes estrechos y de poca altura, sin flujo de corrientes de aire, funcionando así como "trampas" para el calor generado tanto por la descomposición del guano, como por el metabolismo de los individuos que conforman la colonia. Asimismo, también retienen la humedad generada, en gran parte, por pequeñas filtraciones de agua a través de estas secciones. Las alas de $N$. stramineus son muy anchas, siendo la superficie relativamente grande (Jennings et al. 2004; Norberg \& Rayner 1987; Torres-Flores 2005). Además la membrana es muy delgada y, al igual que otros natálidos, se deshidrata rápidamente en ambientes secos, volviéndose rígida y quebradiza. Por ende, podría esperarse que este murciélago pierda mucho calor corporal y humedad en sus alas de forma considerable durante sus actividades de forrajeo, sobre todo si tomamos en cuenta que la temperatura y humedad externas son menores a las encontradas en sus sitios de descanso. Estas razones explicarían por qué prefiere lugares calientes y muy húmedos para descansar, ya que estas condiciones son ideales para ahorrar energía y mantener una temperatura corporal óptima, así como para mantener hidratadas sus alas.

En la cueva "El Salitre", la temperatura y humedad externas registradas durante la noche oscilaron ampliamente y sus valores son, en su gran mayoría, mucho menores a los registrados en las sitios de percha de N. stramineus. Aunque en estos sitios se presentaron diferencias en la temperatura y humedad entre meses, estas condiciones fueron relativamente estables a lo largo del año y siempre $>25^{\circ} \mathrm{C}$ y $>$ $92 \%$ respectivamente, teniendo esta especie una fuerte preferencia por secciones de 
la cueva con dichas características microclimáticas. Las diferencias registradas en estas variables entre el ambiente interno y externo también muestran una de las ventajas que poseen las cuevas como refugio para los murciélagos, esto es, ofrecer resguardo ante variaciones ambientales externas y proporcionar condiciones microclimáticas constantes y adecuadas para su supervivencia (Altringham 1996; Kunz 1982).

En México, esta especie se ha encontrado siempre en refugios con humedad alta (> 74\%) y, en la gran mayoría de los casos, con temperaturas cálidas $\left(25-36.6^{\circ} \mathrm{C} ;\right.$ e.g. Arita \& Vargas 1995; Martínez-Coronel et al. 1996; Sánchez-Hernández et al. 2002; este estudio). No obstante, en algunos sitios se ha encontrado a temperaturas templadas $\left(<25^{\circ} \mathrm{C}\right.$ ) incluso hasta los $16.5^{\circ} \mathrm{C}$ (Ávila-Flores \& Medellín 2002, Vargas-Contreras 1998). En nuestros muestreos también encontramos poblaciones viviendo en refugios con temperaturas templadas ("El Amparo" y "San Sebastián de las Grutas") pero con humedad relativa superior al $80 \%$, ya que en el interior de ambas hay arroyos grandes con agua en circulación. Esto sugiere que $N$. stramineus puede ser tolerante a un rango más amplio de temperatura que de humedad en sus sitios de descanso.

Los datos muestran que $N$. stramineus tiene una gran tendencia a refugiarse con una gran variedad de especies de murciélagos, sobre todo con mormópidos y algunos filostómidos. Los mormópidos son cavernícolas estrictos y forman colonias de varios miles de individuos (Bateman \& Vaughan 1974; Bonaccorso et al. 1992; SilvaTaboada 1979). Los filostómidos G. soricina, D. rotundus y A. jamaicensis aunque no son cavernícolas estrictos a menudo se refugian en cuevas, donde llegan a elegir secciones calientes, aunque de menor temperatura y humedad que las utilizadas por los mormópidos (Arita \& Vargas 1995; Ávila-Flores \& Medellín 2004). Por consiguiente, la alta frecuencia de asociación con estos grupos de murciélagos se debe, en parte, a la misma necesidad en cuanto a condiciones microclimáticas similares, esto es, sitios con temperatura y humedad altas. Asimismo los beneficios termoregulatorios que ofrece el agrupamiento en colonias formadas por muchas especies, posiblemente también promueva esta tendencia a asociarse. A excepción de A. jamaicensis, las 6 especies que se han encontrado asociadas con mayor frecuencia a $N$. stramineus se refugian en la cueva "El Salitre", Colima (López-Wilchis \& Torres-Flores 2005) aunque se encuentran segregadas espacialmente (Fig. 4). Mormoops megallophylla, Pteronotus davyi, P. parnelli y P. personatus utilizan las partes más profundas de la cueva, donde la temperatura es $>27^{\circ} \mathrm{C}$ y la humedad es del $100 \%$, y donde la gran mayoría de sus colonias se albergan en una gran cámara principal. En tanto, G. soricina y D. rotundus ocupan las secciones intermedias donde fluyen pequeñas corrientes de aire, con temperaturas $<25.5^{\circ} \mathrm{C}$ y humedad $<$ $85 \%$. Las diferencias en estas condiciones microclimáticas y probablemente la poca tolerancia interespecífica podrían influir en esta segregación espacial dentro de la cueva. 
En términos generales, $N$. stramineus prefiere albergarse en los lugares más profundos de la cueva, colgándose individualmente en paredes y techos inclinados de poca altura, en condiciones de obscuridad total. Estos hábitos de percha también fueron observados en otros refugios que fueron explorados durante nuestros recorridos y coinciden con observaciones realizadas en otras localidades (Arita \& Vargas 1995; Hall \& Dalquest 1963; Sánchez-Hernández et al. 2002; Taddei \& Uieda 2001; Trajano \& Gimenez 1998). También se han observado algunas colonias perchando en partes altas, por arriba de los $20 \mathrm{~m}$ de la superficie (Jones et al. 1965). Asimismo, se ha encontrado refugiándose en lugares poco profundos y con buena iluminación (Cueva Colotlán, este estudio; Sánchez-Hernández et al. 2002).

Nuestras observaciones indican que este murciélago tiene poca o moderada tolerancia a la presencia humana; en condiciones de obscuridad total o luminosidad tenue los individuos permanecen quietos ante señales de disturbio (voces o pasos), mientras que ante fuentes luminosas directas permanecen alertas, aunque permiten la cercanía por algunos segundos, volando después y albergándose en partes más profundas. Algunos llegamos a verlos ligeramente aletargados, pudiendo aproximarnos a ellos a corta distancia, activándose y emprendiendo el vuelo posteriormente.

La información consignada en el presente estudio puede servir para identificar refugios que requieren ser conservados. Lo anterior es importante ya que como se muestra en los datos recabados, si bien este murciélago es abundante y puede desenvolverse en una gran variedad de hábitats, depende en mucho de la presencia de cuevas o minas con humedad alta y, en general, con temperatura cálida, siendo estas condiciones un requerimiento indispensable para su supervivencia. Además si bien suele formar colonias de cientos o miles de individuos, en varios de sus refugios sus poblaciones han sido afectadas. Esto se constató al visitar algunos lugares donde anteriormente se había documentado y en los que no fueron observados. En otros donde aun permanecen, sufren en la actualidad disturbio constante por las visitas no controladas (e.g. cueva "Los Laguitos", Chiapas); y en otros que han sido severamente modificados por el turismo, es factible que hayan disminuido sus poblaciones o incluso desaparecido (e.g. Grutas de Cacahuamilpa, Guerrero).

AGRADECIMIENTOS. Agradecemos a Armando Vega por su ayuda durante el trabajo de campo en la cueva de estudio y al comisario Carlos Vega por facilitar nuestra estancia en el poblado Los Ortices, Colima. Al Dr. Miguel León Galván y los biólogos Neófito Ángeles Pérez, Luis Martínez Méndez, Dafne Zárate Martínez e Iliana Pérez Espinoza por su ayuda en el trabajo de campo durante los recorridos a diferentes estados del país. Al Dr. Arnulfo Moreno por su ayuda en el trabajo de campo y las facilidades otorgadas durante los recorridos en el estado de Tamaulipas. Al Dr. Joaquín Arroyo Cabrales por facilitar algunas de las referencias revisadas en este trabajo. El trabajo de campo fue financiado con apoyos otorgados a RLW por la Universidad Autónoma 
Metropolitana y el Consejo Nacional de Ciencia y Tecnología (CONACyT) y por Idea Wild a JWTF. El primer autor está inscrito en el Programa de Doctorado de Ciencias Biológicas de la Universidad Autónoma Metropolitana y recibió una beca para estudios de posgrado (No. 172705) por parte del CONACyT.

\section{LITERATURA CITADA}

Akins, J. B., M. L. Kennedy, G. D. Schnell, C. Sánchez-Hernández, M. L. Romero-Almaraz, M. C. Wooten \& T. L. Best. 2007. Flight speeds of three species of Neotropical bats: Glossophaga soricina, Natalus stramineus, and Carollia subrufa. Acta Chiropterologica, 9:477-482.

Altringham, J. D. 1996. Bats: biology and behavior. Oxford University Press, Oxford.

Arita, H. T. 1993. Conservation biology of the cave bats of Mexico. Journal of Mammalogy, 74:693702.

Arita, H. T. \& J. A. Vargas. 1995. Natural history, interspecific association, and incidence of cave bats of Yucatan, Mexico. Southwestern Naturalist, 40:29-37.

Ávila-Flores, R. \& R. A. Medellín. 2004. Ecological, taxonomic, and physiological correlates of cave use by Mexican bats. Journal of Mammalogy, 85:675-687.

Bateman, G. C. \& T. A. Vaughan. 1974. Nightly activities of mormoopid bats. Journal of Mamma$\log y$, 55:45-65.

Baudinette, R.V., S. K. Churchill, K. A. Christian, J. E. Nelson \& P. J. Hudson. 2000. Energy, water balance and the roost microenvironment in three Australian cave-dwelling bats (Microchiroptera). Journal of Comparative Physiology B, 170:439-446.

Bonaccorso, F. J., A. Arends, M. Genoud, D. Cantoni \& T. Morton. 1992. Thermal ecology of moustached and ghost-faced bats (Mormoopidae) in Venezuela. Journal of Mammalogy, 73:365-378.

CNMA. 2007. Colección Nacional de Mamíferos. Portal UNIBIO Instituto de Biología, Universidad Nacional Autónoma de México. http://unibio.unam.mx/ (consultada el 10/10/2007).

Dalquest, W. W. 1950. The genera of the chiropteran family Natalidae. Journal of Mammalogy, 31:436-443.

Emmons, L. H. 1997. Neotropical rainforest mammals: a field guide. Second edition. University of Chicago Press, Chicago.

Goodwin, G. G. 1959. Bats of the subgenus Natalus. American Museum Novitates, 1977:1-22.

Graham, G. L. 1988. Interspecific associations among Peruvian bats at diurnal roost and roost sites. Journal of Mammalogy, 69:711-720.

Hall, E. R. \& W. W. Dalquest. 1963. The mammals of Veracruz. University of Kansas Publications, Museum of Natural History, 14:165-362.

INEGI. 2009. Instituto Nacional de Estadística, Geografía e Informática. México. www.inegi.gob.mx (Consultada 10/03/2009).

Jennings, N. V., S. Parsons, K. E. Barlow \& M. R. Gannon. 2004. Echolocation calls and wing morphology of bats from the West Indies. Acta Chiropterologica, 6:75-90.

Jones, J. K. Jr., J. D. Smith \& T. Álvarez. 1965. Notes on bats from the Cape Region of Baja California. Transactions of the San Diego Society of Natural History, 14:53-56.

Kerth, G., K. Weissmann \& B. König. 2001. Day roost selection in female Bechstein's bat (Myotis bechsteinii): a field experiment to determine the influence of roost temperature. Oecologia, 126:1-9.

Krutzch, P. H. 2000. Anatomy, physiology and cyclicity of the male reproductive tract. Pp. 91-155 In: E. G. Crichton and P. H. Krutzch (Eds.). Reproductive biology of bats. Academic Press, London.

Kunz, T. H. 1982. Roosting ecology of bats. Pp. 1-55 In: T. H. Kunz (Ed.). Ecology of bats. Plenum Press, New York. 
Torres-Flores \& López Wilchis: Condiciones microclimáticas en refugios de Natalus stramineus

López-Wilchis, R. 2003. Base de datos de los mamíferos de México depositados en colecciones de Estados Unidos y Canadá. Universidad Autónoma Metropolitana-Iztapalapa. (http://investigacion. izt.uam.mx/mamiferos/)

López-Wilchis, R. \& J. W. Torres-Flores. 2005. Especies de murciélagos presentes en la cueva "El Salitre", Colima, México. Vertebrata Mexicana, 17:7-12.

Martínez-Coronel, M., M. G. Pérez \& J. P. Albores. 1996. Los murciélagos de la cueva "Los Laguitos", su importancia biológica y social. Investigación, Ciencias y Artes en Chiapas, 1:10-18.

Medellín, R., H. T. Arita \& O. Sánchez. 1997. Identificación de murciélagos de México. Clave de campo. Publicaciones Especiales 2. Asociación Mexicana de Mastozoología, A. C., México, D. F.

Mitchell, G. C. 1967. Population study of the funnel-eared bat (Natalus stramineus) in Sonora. Southwestern Naturalist, 12:172-175.

Norberg, U. M. \& J. M. V. Rayner. 1987. Ecological morphology and flight in bats (Mammalia: Chiroptera): wing adaptations, flight performance, foraging strategy and echolocation. Philosophical Transactions of the Royal Society of London, Series B, 316:335-427.

Ramírez-Pulido, J., J. Arroyo-Cabrales \& A. Castro-Campillo. 2005. Estado actual y relación nomenclatural de los mamíferos terrestres de México. Acta Zoológica Mexicana (n. s.), 21:21-82.

Reid, F. 1997. A field guide to the mammals of Central America and Southeast Mexico. Oxford University Press, New York.

Rodríguez-Durán, A. 1998. Nonrandom aggregations and distribution of cave-dwelling bats in Puerto Rico. Journal of Mammalogy, 79:141-146.

Rodríguez-Durán, A. \& J. A. Soto-Centeno. 2003. Temperature selection by tropical bats roosting in caves. Journal of Thermal Biology, 28:465-468.

Rydell, J., H. T. Arita, M. Santos \& J. Granados. 2002. Acoustic identification of insectivorous bat (Order Chiroptera) of Yucatan, Mexico. Journal of Zoology, 257:27-36.

Sánchez-Hernández, C., M. L. Romero-Almaraz \& M. A. Gurrola-Hidalgo. 2002. Natalus stramineus saturatus (Dalquest y Hall, 1949). Pp. 403-405 In: F. A. Noguera, J. H. Vega-Rivera, A. N. García-Aldrete y M. Quesada-Avendaño (Eds.). Historia Natural de Chamela. Instituto de Biología, Universidad Nacional Autónoma de México, México, D. F.

Silva-Taboada, G. 1979. Los murciélagos de Cuba. Editorial Academia, La Habana.

Simmons, N. B. 2005. Order Chiroptera. Pp. 312-529 In: D. E. Wilson and D. M Reeder (Eds.). Mammal species of the World: a taxonomic and geographic reference. Third edition. Johns Hopkins University Press, Baltimore.

Speakman, J. R. \& D. W. Thomas. 2003. Physiological ecology and energetics of bats. Pp. 430-492 In: T. H. Kunz and M. B. Fenton (Eds.). Bat Ecology. University of Chicago Press, Chicago.

StataCorp. 2003. Stata: statistical software for professionals. Release 8.0 [computer program]. Stata Corporation, College Station, Texas.

Taddei, V. A. \& W. Uieda. 2001. Distribution and morphometrics of Natalus stramineus from South America (Chiroptera, Natalidae). Iheringia, Serie Zoologia, 91:123-132.

Tejedor, A. 2005. A new species of funnel-eared bat (Natalidae: Natalus) from Mexico. Journal of Mammalogy, 86:1109-1120.

Tejedor, A. 2006. The type locality of Natalus stramineus (Chiroptera: Natalidae): implications for the taxonomy and biogeography of the genus Natalus. Acta Chiropterologica, 8:361-380.

Tejedor, A., V. Da C. Tavares \& G. Silva-Taboada. 2005. A revision of extant Greater Antillean bats of the genus Natalus (Chiroptera: Natalidae). American Museum Novitates, 3493:1-22.

Torres-Flores, J. W. 2005. Estructura de una comunidad tropical de murciélagos presente en la cueva “El Salitre”, Colima, México. Tesis de Maestría, Universidad Autónoma Metropolitana, Unidad Iztapalapa. México, D. F. 
Trajano, E. \& E. A. Gimenez. 1998. Bat community in a cave from eastern Brazil, including a new record of Lionycteris (Phyllostomidae, Glossophaginae). Studies on Neotropical Fauna and Environment, 33:69-75.

Tuttle, M. D. \& D. Stevenson. 1982. Growth and survival of bats. Pp. 105-150 In: T. H. Kunz (Ed.). Ecology of bats. Plenum Press, New York.

Vargas-Contreras, J. A. 1998. Factores microclimáticos y selección del refugio diurno por murciélagos cavernícolas en Gómez Farías, Tamaulipas. Tesis de Maestría, Facultad de Ciencias, Universidad Nacional Autónoma de México. México, D. F. 
Torres-Flores \& López Wilchis: Condiciones microclimáticas en refugios de Natalus stramineus






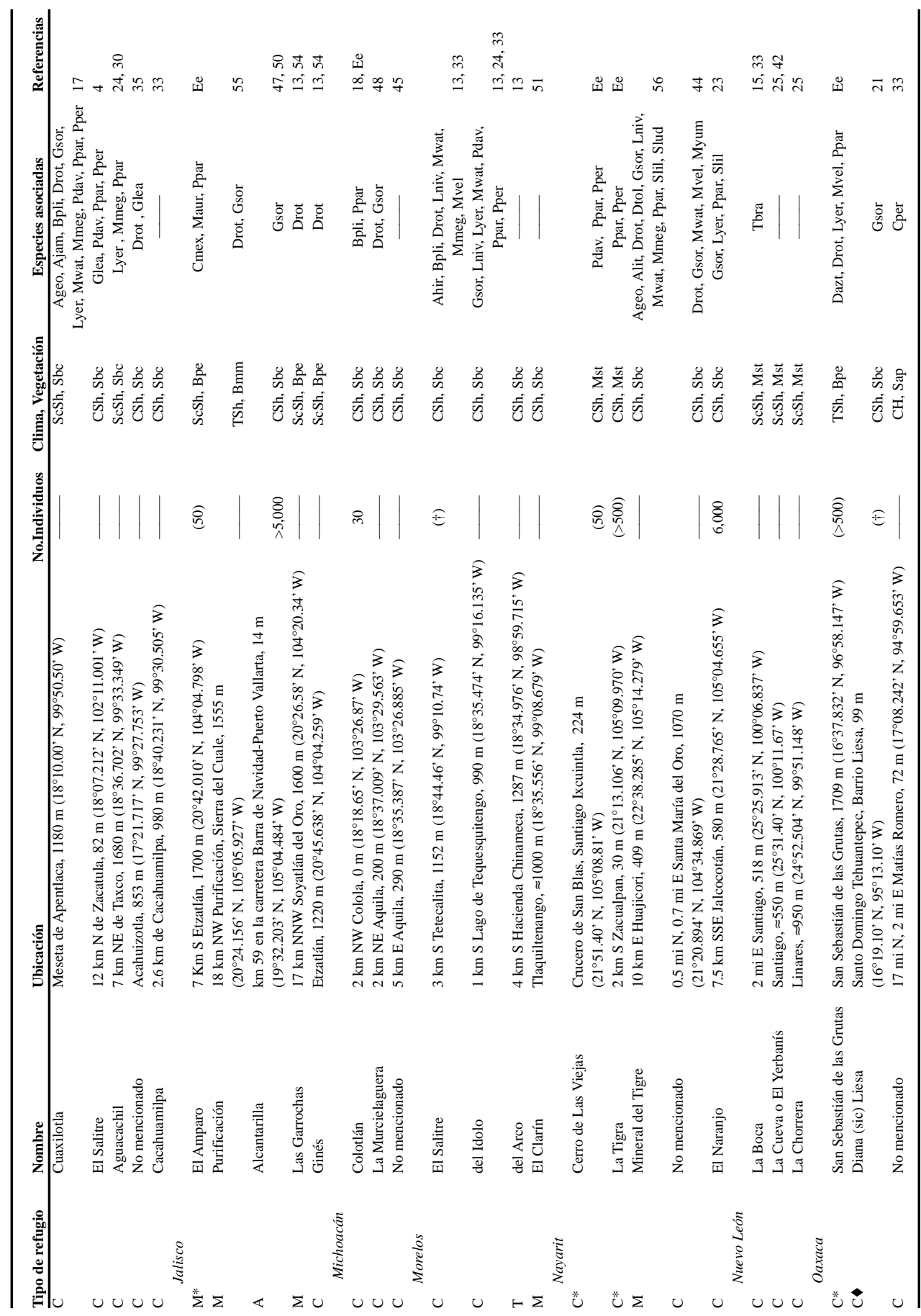


Torres-Flores \& López Wilchis: Condiciones microclimáticas en refugios de Natalus stramineus

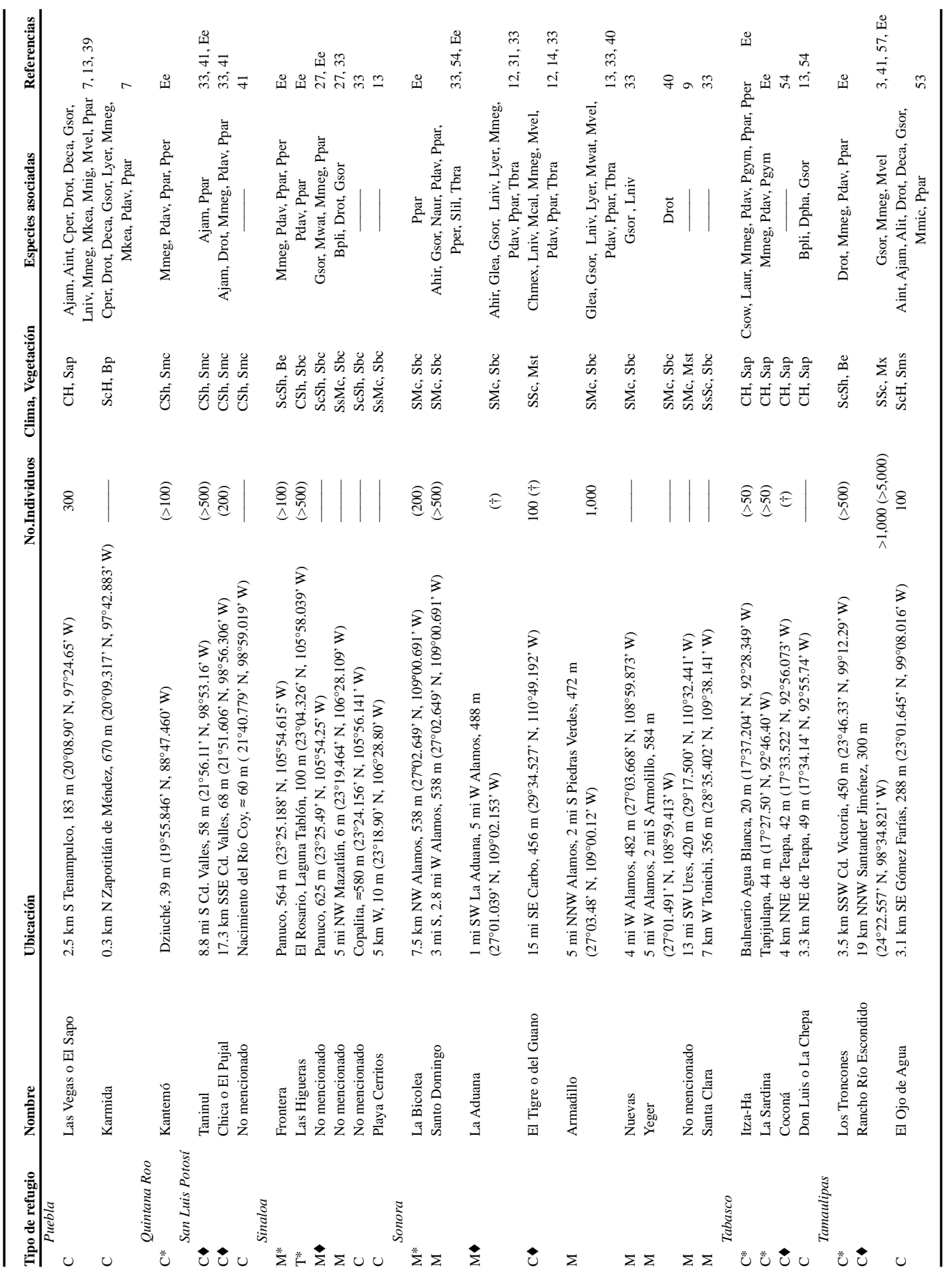




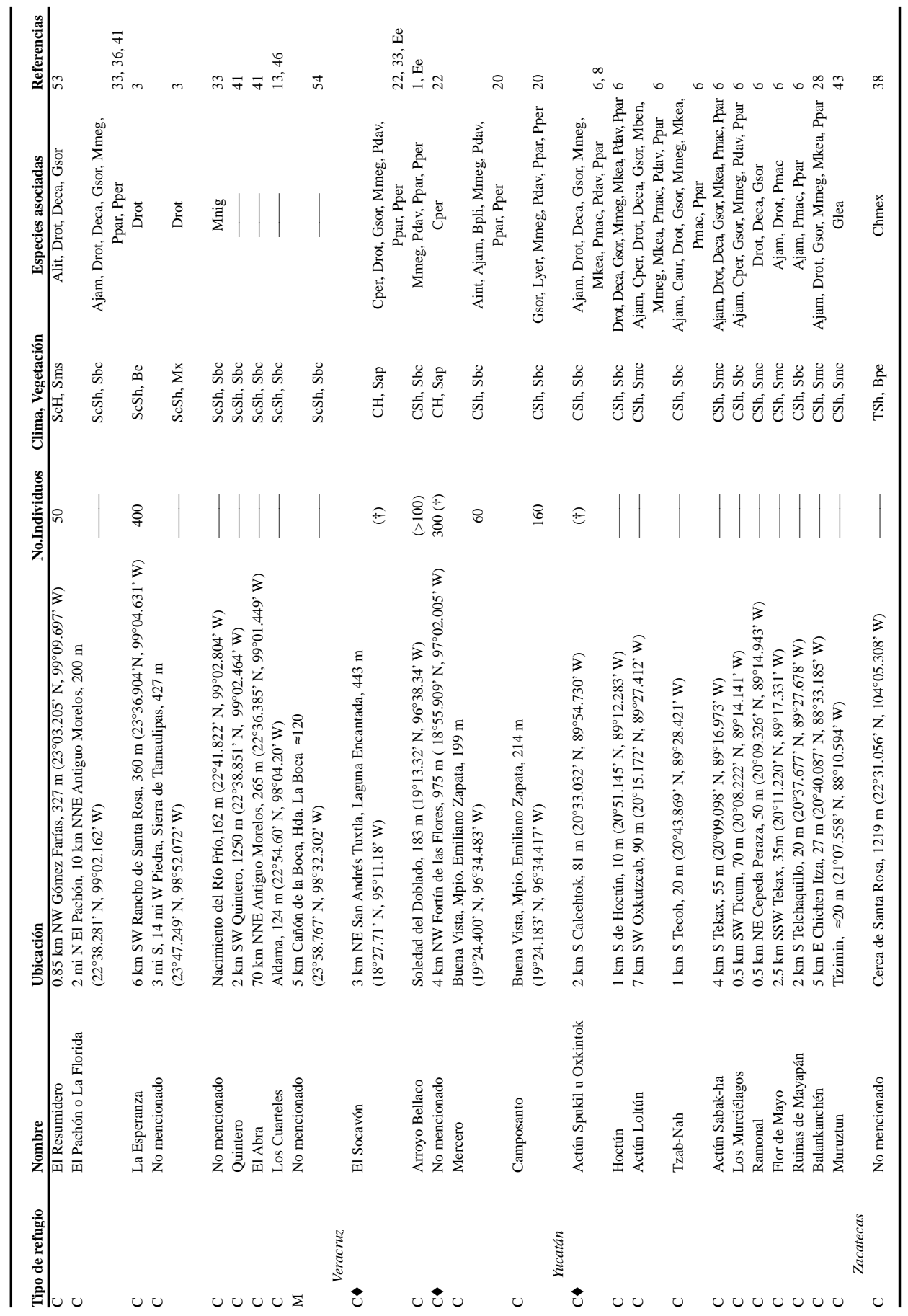


Torres-Flores \& López Wilchis: Condiciones microclimáticas en refugios de Natalus stramineus

Tipo de refugio: $\mathrm{C}=$ cueva o gruta, $\mathrm{M}=$ mina, $\mathrm{T}=$ túnel, $\mathrm{A}=$ alcantarilla

- Refugios con más de 30 años de antigüedad de haber sido registrados y considerados importantes por su posición geográfica y/o por el número de organismos presentes.

* Sitios donde se registra por primera vez a $N$. stramineus.

$(\approx)$ Número máximo de individuos estimado por nosotros.

$(\dagger$ Sitios donde se ha reportado previamente la presencia de N. stramineus pero que durante los muestreos realizados por nosotros no se encontraron individuos.

Clima: $\mathrm{CH}=$ Cálido-húmedo, $\mathrm{CSh}=$ Cálido-subhúmedo, $\mathrm{ScH}=$ Semicálido-húmedo, $\mathrm{ScSh}=$ Semicálidosubhúmedo, $\mathrm{SfSh}=$ Semifrío-subhúmedo, TSh = Templado-subhúmedo, MsSc = Muy seco-semicálido, SMc $=$ Secomuy cálido, $\mathrm{SSc}=$ Seco-semicálido, $\mathrm{ScMc}=$ Semiseco-muy cálido, $\mathrm{SsSc}=$ Semiseco-semicálido.

Vegetación circundante: $\mathrm{Bp}=$ Bosque de pino, $\mathrm{Be}=\mathrm{Bosque}$ de encino, $\mathrm{Bpe}=$ Bosque de pino-encino, $\mathrm{Bmm}=$ Bosque mesófilo de montaña, Mst= Matorral subtropical espinoso, $\mathrm{Mx}=$ Matorral xerofito, $\mathrm{Sbc}=$ Selva baja caducifolia, $\mathrm{Smc}=$ Selva mediana caducifolia, $\mathrm{Sms}=$ Selva mediana subcaducifolia, $\mathrm{Sap}=$ Selva alta perennifolia, Smp $=$ Selva mediana perennifolia.

Especies asociadas: Ageo $=$ Anoura geoffroyi, Ahir $=$ Artibeus hirsutus, Aint $=$ Artibeus intermedius, Ajam $=$ Artibeus jamaicensis, Alit $=$ Artibeus lituratus, $\mathrm{Bpli}=$ Balantiopteryx plicata, Cper $=$ Carollia perspicillata, Csow $=$ Carollia sowelli, $\mathrm{Csub}=$ Carollia subrufa, $\mathrm{Chmex}=$ Choeronycteris mexicana, Caur $=$ Chrotopterus auritus, $\mathrm{Cmex}$ $=$ Corynorhinus mexicanus, Dazt $=$ Dermanura azteca , Dpha $=$ Dermanura phaeotis, Dtol $=$ Dermanura tolteca, Drot $=$ Desmodus rotundus, Deca $=$ Diphylla ecaudata, $\mathrm{Glea}=$ Glossophaga leachii,, $\mathrm{Gmor}=$ Glossophaga morenoi, Gsor = Glossophaga soricina, Gsyl = Glyphonycteris sylvestris, Lyer $=$ Leptonyceris yerbabuenae, Lniv $=$ Leptonycteris nivalis, Laur = Lonchorhina aurita, $\mathrm{Mcal}=$ Macrotus californicus, Mwat $=$ Macrotus waterhousii, Mmic = Micronycteris microtis, Mben = Mimon bennettii, Mmeg = Mormoops megalophylla, Maur $=$ Myotis auriculus, Mkea = Myotis keaysi, Mnig = Myotis nigricans, $\mathrm{Mvel}=$ Myotis velifer , Myum = Myotis yumanensis, Naur $=$ Nyctinomops aurispinosus, Nlat $=$ Nyctinomops laticaudatus, Phes $=$ Parastrellus hesperus, Pmac $=$ Peropteryx macrotis, Pdav = Pteronotus davyi, Pgym = Pteronotus gymnonotus, Ppar $=$ Pteronotu parnellii, Pper $=$ Pteronotus personatus, Slil = Sturnira lilium, Slud $=$ Sturnira ludovici, Tbra $=$ Tadarida brasiliensis .

Referencias: Ee = Este estudio. 1. Aguilar \& Ruiz-C. 1995. Tesis de Licenciatura, Univ. Nac. Aut. Méx. México, D. F. 2. Akins et al. 2007. Act. Chirop., 9:477-482. 3. Álvarez. 1963. Univ. Kansas Publ. Mus. Nat. Hist., 14:363473. 4. Álvarez. 1968. Rev. Soc. Mex. Hist. Nat., 29:21-35. 5. Anderson. 1972. Bull. Amer. Mus. Nat. Hist., 148:149410. 6. Arita \& Vargas. 1995. South. Nat., 40:29-37. 7. Ávila-Flores \& Medellín. 2004. J. Mamm., 85:675-687. 8. Birney et al. 1974. Occas. Pap. Bell Mus. Nat. Hist., 13:1-25. 9. Broadbooks. 1961. J. Mamm., 42:403. 10. Caballero. 1942. An. Inst. Biol. Univ. Nac. Aut. Méx. Ser. Zool., 13:105-109. 11. Cicourel. 2003. Tesina de Licenciatura. Univ. Aut. Metr. México, D. F. 12.-Cockrum \& Bradshaw. 1963. Amer. Mus. Nov., 2138:1-9. 13. CNMA. 2007. Colección Nacional de Mamíferos, Portal UNIBIO. Univ. Nac. Aut. Méx. http://unibio.unam.mx/ (consultada el 10/10/2007). 14. Constantine. 1959. J. Mamm., 40:442. 15. Davis \& Carter. 1962. South. Nat. 7:64-74. 16. Escalona-Segura et al. 2002. Rev. Mex. Masto., 6:99-103. 17. Galicia. 2004. Tesis de Licenciatura, Univ. Nac. Aut. Méx. México, D. F. 18. García. 2002. Tesina de Licenciatura, Univ. Aut. Metr. México, D. F. 19. González-Ruiz et al. 2004. Pp. 53-82. In: A. Castro-Campillo y J. Ortega (eds.). Homenaje a la trayectoria mastozoológica de José Ramírez Pulido. Univ. Aut. Metr. México, D. F. 20. Gonzalez-Christen (datos no publicados). 21. Goodwin. 1969. Bull. Amer. Mus. Nat. Hist. 141:1-270. 22. Hall \& Dalquest. 1963. Univ. Kansas Publ. Mus. Nat. Hist., 14:165-362. 23. Hernández. 2000. Tesis de Licenciatura. Univ. Nac. Aut. Méx. México, D. F. 24. Hoffmann et al. 1986. Manual de Bioespeleología. Univ. Nac. Aut. Méx. México, D. F. 25. Jiménez-Guzmán et al. 1999. Mamíferos de Nuevo León. Univ. Aut. Nuevo León. 26. Jones et al. 1965. Trans. San Diego Soc. Nat. Hist., 14:53-56. 27. Jones et al. 1972. Occas. Pap. Mus. Nat. Hist., Univ. Kansas, 6:1-29. 28. Jones et al. 1973. Occas. Pap. Mus. Texas Tech Univ., 13:1-31. 29. Knobloch. 1942. J. Mamm., 23:297-298. 30. León-Paniagua \& Romo. 1993. Pp. 45-64 In: R. A. Medellín y G. Ceballos (eds.). Avances en el estudio de los mamíferos de México. Asoc. Mex. Mast. México, D. F. 31. Loomis \& Davis. 1965. J. Mamm., 46:497. 32. López-González \& García-Mendoza. 2006. Act. Zool. Mex. (n.s.). 22:109-135. 33. López-Wilchis, R. 2003. Base de datos de los mamíferos de México depositados en colecciones de Estados Unidos y Canadá. Univ. 
Aut. Metro. (http://investigacion.izt.uam.mx/mamiferos/) 34. López-Wilchis \& Torres-Flores. 2005. Vert. Mex., 17:712. 35. Lukens \& Davis. 1957. J. Mamm., 38:1-14. 36. Martin \& Martin. 1954. J. Mamm., 35:584-585. 37. MartínezCoronel et al. 1996. Inv. Cien. Art. Chiapas 1:10-18. 38. Matson \& Patten. 1975. Contri. Sci. Nat. Hist. Mus. Los Angeles County, 263:1-12. 39. Medellín \& López-Forment. 1986. An. Inst. Biol. Univ. Nac. Aut. Méx. Ser. Zool., 56:1027-1034. 40. Mitchell. 1967. South. Nat. 12:172-175. 41. Mollhagen. 1971. Assoc. Mex. Cav. Stud. Bull., 4:1922. 42. Moreno-Valdez. 1998. Rev. Mex. Masto., 3:5-25. 43. Pearse \& Kellogg. 1938. Carnegie Inst. Washington Publ., 491:301-304. 44. Polaco et al. 1992. Texas Jour. Sci., 44:331-338. 45. Polaco \& Muñiz-Martínez. 1987. An. Esc. Nac. Cien. Biol., 31:63-89. 46. Rudnick. 1960. Univ. California Publ. of Entom., 17:157-284. 47. SánchezHernández et al. 1979. An. Inst. Biol. Univ. Nac. Aut. Méx. Ser. Zool., 50:787. 48. Sánchez-Hernández et al. 1985. J. Mamm., 66:549-553. 49. Sánchez-Hernández \& Romero-A. 1995. Cuadernos del Instituto de Biología, No. 24, Univ. Nac. Aut. Méx. México, D. F. 50. Sánchez-Hernández et al. 2002. Pp. 403-405. In: F. A. Noguera, J. H. Vega Rivera, A. N. García-Aldrete y M. Quesada Avendaño (eds.). Historia Natural de Chamela. Univ. Nac. Aut. Méx. México, D. F. 51. Taylor et al. 1999. J. Trop. Med. Hyg., 61:914-919. 52. Torres-Flores. 2005. Tesis de Maestría. Univ. Aut. Metr. México, D. F. 53. Vargas-Contreras. 1998. Tesis de Maestría. Univ. Nac. Aut. Méx. México, D. F. 54. Villa-R. B. 1966. Los murciélagos de México. Univ. Nac. Aut. Mex. México, D. F. 55. Watkins et al. 1972. Spec. Pub. Mus. Texas Tech Univ. 1:1-44. 56. Webb et al. 1981. An. Inst. Biol. Univ. Nac. Aut. Mex. Ser. Zool., 51:699-702. 57. Wilson et al. 1985. Act. Zool. Mex. (n.s.), 8:1-26. 